\title{
Valor Nutritivo de Alguns Alimentos para Rãs
}

\section{Josevane Carvalho Castro1, Dório Anderson Vicente da Silva², Rondnelly Bitencourt Santos², Vinícius Fassarella Modenesi ${ }^{2}$, Eduardo Faé de Almeida ${ }^{2}$}

RESUMO - O objetivo deste trabalho foi determinar o valor nutritivo de alguns alimentos utilizados em rações de rãs, em um ensaio de digestibilidade, utilizando-se o método de alimentação forçada, com 84 rãs na fase de terminação ( $128,5 \mathrm{~g} \pm 2 \mathrm{~g})$ e 120 rãs na fase inicial (27,3 g $\pm 2 \mathrm{~g})$, distribuídas em gaiolas de metabolismo. Quarenta rãs na fase inicial e 28 na fase de terminação permaneceram de jejum, para determinar as perdas endógenas e metabólicas. Os valores de matéria seca aparentemente metabolizável (MSAM - \%), energia metabolizável aparente (EMA - kcal/kg), energia metabolizável aparente corrigida pela retenção de nitrogênio (EMAn - kcal $/ \mathrm{kg}$ ), energia metabolizável verdadeira (EMV - kcal/kg) e energia metabolizável verdadeira corrigida pela retenção de nitrogênio (EMVn - kcal/kg) determinados para os diferentes ingredientes, com rãs na fase inicial foram, respectivamente: fubá de milho 55,70; 1842;1798; 1948 e 1896; amido de milho 49,60; 1553; 1524; 1634 e 1599; farelo de trigo 35,95; 1192; 1097; 1361 e 1256; farelo de soja 76,97; 2856; 2405; 2965 e 2508; farinha de peixe 55,16; 2288; 1915; 2386 e 1995 e farinha de carne e ossos 29,63; 1683; 1418; 1765 e 1495 . Os valores de MSAM (\%), EMA (kcal/kg), EMAn (kcal/kg), EMV (kcal/kg) e EMVn (kcal/kg) determinados com rãs na fase de terminação, para os diferentes ingredientes, foram, respectivamente: fubá de milho, 58,44; 1934; 1893; 1971 e 1827; amido de milho, 73,03; 2132; $2085 ; 2175$ e 2007; farelo de trigo, 87,54; 2800; 2644; 2838 e 2602; farelo de soja, 71,50; 2718; 2284; 2754 e 2220; farinha de peixe, 60,24; 2266; $1817 ; 2347$ e 1672; e farinha de carne e ossos, 59,01; 2080; 1835; 2113 e 1797.

Palavras-chave: digestibilidade, energia metabolizável, rã touro, Rana catesbeiana

\section{Nutritive Values of Some Frog Feeds}

\begin{abstract}
The objective of this study was to determine the nutritive values of some feeds from frogs diets, in a digestibility assay, using the forced feeding method, with 84 frogs at finishing stage $(128,5 \mathrm{~g} \pm 2 \mathrm{~g})$ and 120 at initial stage $(27,3 \mathrm{~g} \pm 2 \mathrm{~g})$, housed in metabolism cages. Forty frogs at initial stage and 28 at finishing stage were fasting, to determine the endogenous and metabolic losses. The values of apparent metabolizable dry matter (AMDM - \%), apparent metabolizable energy (AME - kcal $/ \mathrm{kg}$ ), apparent metabolizable energy corrected by the nitrogen retention (AMEn - kcal/kg), true metabolizable energy (TME - kcal/kg) and true metabolizable energy corrected by the nitrogen retention (TMEn - kcal $/ \mathrm{kg}$ ) determined for the different ingredients, with frogs at the initial stage were: corn meal 55.70; 1842; 1798; 1948 and 1896; corn starch 49.60; 1553; 1524; 1634 and 1599; wheat meal 35.95; 1192; $1097 ; 1361$ and 1256 ; soybean meal 76.97; 2856; 2405; 2965 and 2508; fish meal 55.16;2288; 1915; 2386 and 1995 and meat and bone meal 29.63; 1683; 1418; 1765 and 1495, respectively. The values of $\operatorname{AMDM}(\%), \operatorname{AME}(\mathrm{kcal} / \mathrm{kg}), \operatorname{AMEn}(\mathrm{kcal} / \mathrm{kg}), \mathrm{TME}(\mathrm{kcal} / \mathrm{kg})$ and TMEn $(\mathrm{kcal} / \mathrm{kg}) \mathrm{determined}$ withy frog at the finishing stage, for the different ingredients, were,: corn meal 58.44; 1934; 1893; 1971 and 1827 ; corn starch 73.03; $2132 ; 2085 ; 2175$ and 2007; wheat meal 87.54; 2800; 2644; 2838 and 2602; soybean meal 71.50; 2718; 2284; 2754 and 2220 ; fish meal $60.24 ; 2266 ; 1817 ; 2347$ and 1672 and meat and bone meal 59.01; 2080; 1835; 2113 and 1797, respectively.
\end{abstract}

Key Words: bullfrog, digestibility, metabolizable energy, Rana catesbeiana

\section{Introdução}

Considerando a importância de se estabelecer um programa de alimentação para as espécies em cultivo, como é o caso da rã-touro, torna-se necessário conhecer sua biologia para se chegar a um manejo mais adequado e fornecer uma alimentação mais ajustada às exigências do animal, para assegurar o menor custo de produção, que, no caso específico da rã, a ração corresponde a $57,1 \%$ do custo total de produção (LIMA e AGOSTINHO, 1992).

A rã touro, assim como todos os anfíbios, é carnívora na fase pós-metamórfica e, assim, geralmente, exige maiores teores de proteína na ração em relação àqueles que têm outros hábitos alimentares (REEDER, 1964 ).

Uma vez que a alimentação e nutrição das rãs não têm uma definição concreta, o grande desafio na ranicultura tem sido o estabelecimento das exigências nutricionais, bem como o manejo da alimentação,

\footnotetext{
1 Doutor em Zootecnia - Professor do Departamento de Zootecnia e Economia Rural do CCA - UFES, Caixa Postal 16 - Alegre, ES. E.mail: jccastro@npd.ufes.br

2 Aluno do curso de Graduação. Bolsista do PIBIC/UFES/CNPq.
} 
para se obter um programa de arraçoamento adequado e, conseqüentemente, maior rentabilidade na atividade. Até o momento, os poucos trabalhos encontrados sobre exigências nutricionais das rãs estão relacionados com exigências de proteína bruta (MONTEIRO et al., 1988; BARBALHO, 1991; MAZZONI et al., 1992a; MAZZONI et al., 1992b; STÉFANI, 1995.

Segundo STÉFANI (1996), o carboidrato em alta concentração na ração (40 a $45 \%$ ) é uma fonte de energia disponível para a rã touro, mostrando um efeito economizador de proteína para o desenvolvimento corporal das rãs.

Hoje, na formulação de rações balanceadas para rãs, costuma-se usar dados de composição proximal e energia metabolizável dos alimentos encontrados em tabelas para aves, como a de ROSTAGNO et al (1988), e/ou peixes, como NATIONAL RESEARCH COUNCIL - NRC (1983). Estes resultados encontrados com peixes e aves, quando extrapolados para rãs, podem acarretar erros, pelo fato de serem animais de espécies diferentes.

LIMA e AGOSTINHO (1988) encontraram uma relação inversa para o consumo de ração em relação ao peso dos animais, variando de $5 \%$, para os animais em terminação, a $12 \%$ do peso vivo, para os animais mais jovens, sendo que para os reprodutores o consumo é de 3 a $5 \%$ do peso vivo.

Vários experimentos foram realizados utilizando-se a técnica da alimentação forçada, com o objetivo de adaptar esta técnica, utilizada por SIBBALD (1976) em aves, para rãs. Foi observado que a determinação da digestibilidade dos alimentos deve ser feita separadamente, de acordo com a idade das rãs; o sexo não influi na digestibilidade dos alimentos para rã touro (CASTRO et al, 1995a); o tempo de passagem do alimento pelo trato digestivo da rã touro independe do número de refeições fornecidas (CASTRO et al, 1995b); e o tempo de jejum mínimo para se efetuar a alimentação forçada é de 24 horas (CASTRO et al, 1995c).

BRAGA et al (1998) e CASTRO et al (1998), utilizando a alimentação forçada, determinaram os valores energéticos de alguns alimentos utilizados em rações de rãs, por intermédio de ensaios de digestibilidade, e concluíram que o valor de energia metabolizável aparente é idêntico ao valor de energia metabolizável verdadeira destes ingredientes, mostrando que a perda endógena, nas rãs, é mínima ou quase nenhuma.

O objetivo deste trabalho foi determinar o valor da matéria seca aparentemente metabolizável, energia metabolizável aparente, energia metabolizável verdadeira, energia metabolizável aparente e energia metabolizável verdadeira corrigidas pela retenção de nitrogênio, de seis ingredientes utilizados em rações de rãs, nas fases inicial e de terminação.

\section{Material e Métodos}

O experimento foi realizado no ranário Experimental do Centro de Ciências Agrárias da Universidade Federal do Espírito Santo (CCA-UFES). Foram utilizados 120 animais na fase inicial, com peso médio de 27,3 g \pm 2 g (imagos), e 84 na fase de terminação, com peso médio de 128,5 g $\pm 2 \mathrm{~g}$ (adultos), alojados em gaiolas de metabolismo para rãs. A gaiola fabricada em fibra de vidro, medindo $30 \times 50 \mathrm{~cm}$, é composta por uma tampa com vários furos, para melhor arejamento interno, com um degrau na parte frontal, onde é colocada a água, sendo que no fundo contém um cano de $1 / 2$ polegada, por onde é retirada a água juntamente com as fezes (Figura 1). As gaiolas foram distribuídas em uma bancada de alvenaria no interior de uma sala com temperatura ambiente controlada entre 25 e $27^{\circ} \mathrm{C}$ e $500 \mathrm{~mL}$ de água, com a finalidade de garantir a umidade para as rãs.

Foram utilizadas três repetições para cada tratamento, em que cada gaiola contendo dez imagos ou sete adultos era considerada uma unidade experimental.

Foi adotado o método da alimentação forçada proposto por CASTRO (1996) e BRAGA (1996), utilizando-se os seguintes ingredientes: fubá de milho, amido de milho, farelo de soja, farelo de trigo, farinha de peixe e farinha de carne e ossos. A composição dos alimentos encontra-se na Tabela 1 . As rãs foram mantidas por 48 horas em jejum nas gaiolas de

Tabela 1 - Composição proximal e valores de energia bruta (EB) dos alimentos na matéria natural

Table 1 - Proximal composition and values of gross energy (GE) of feeds, as fed basis

\begin{tabular}{|c|c|c|c|}
\hline $\begin{array}{l}\text { Ingredientes } \\
\text { Ingredients }\end{array}$ & $\begin{array}{l}\mathrm{EB}(\mathrm{kcal} / \mathrm{kg}) \\
G E(k c a l / k g)\end{array}$ & $\begin{array}{l}\mathrm{PB}(\%) \\
C P(\%)\end{array}$ & $\begin{array}{l}\operatorname{DM}(\%) \\
M S(\%)\end{array}$ \\
\hline $\begin{array}{l}\text { Fubá de milho } \\
\text { Corn meal }\end{array}$ & 3794 & 8,26 & 86,17 \\
\hline $\begin{array}{l}\text { Farelo de soja } \\
\text { Sovbean meal }\end{array}$ & 3933 & 43,84 & 89,37 \\
\hline $\begin{array}{l}\text { Farelo de trigo } \\
\text { Wheat meal }\end{array}$ & 3925 & 15,70 & 88,37 \\
\hline $\begin{array}{l}\text { Amido de milho } \\
\text { Corn starch }\end{array}$ & 3115 & 6,47 & 85,58 \\
\hline $\begin{array}{l}\text { Farinha de peixe } \\
\text { Fish meal }\end{array}$ & 3611 & 51,62 & 94,86 \\
\hline $\begin{array}{l}\text { Farinha de carne e ossos } \\
\text { Meat and bone meal }\end{array}$ & 3110 & 37,70 & 92,03 \\
\hline
\end{tabular}




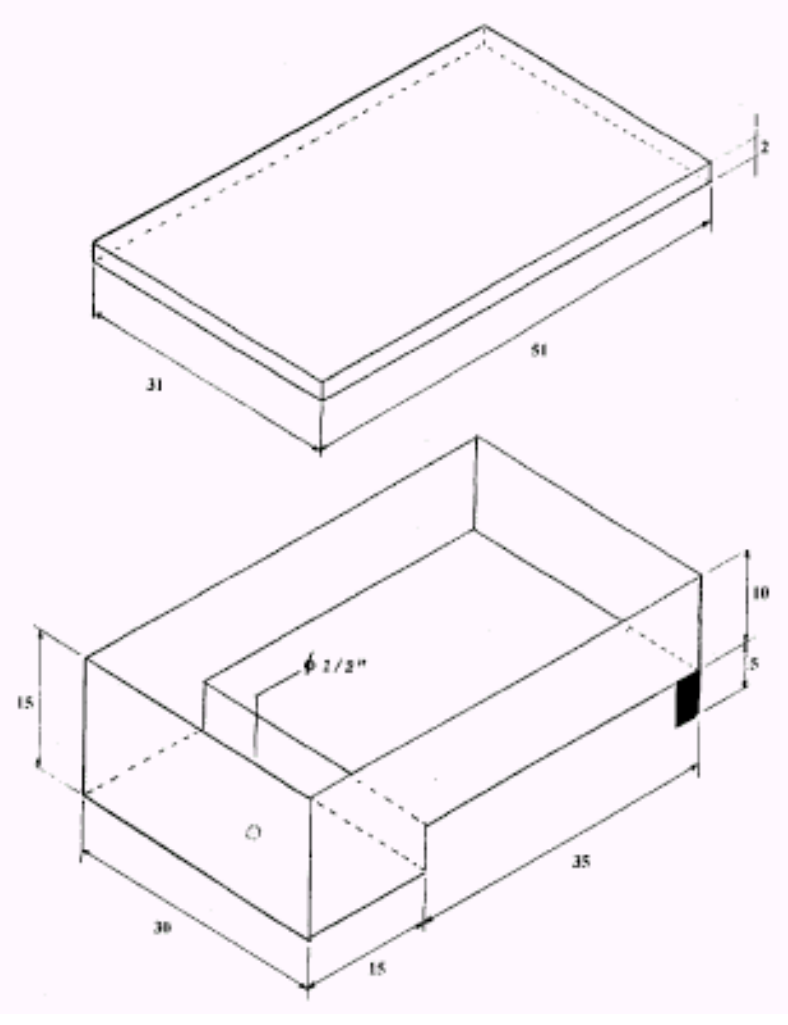

Figura 1 - Caixa de metabolismo para rãs (medidas em cm). Figure 1 - Metabolism box for frogs (size in $\mathrm{cm}$ ).

metabolismo para o esvaziamento do tubo digestivo. Em seguida, foi feita a alimentação forçada do alimento teste, fornecendo uma quantidade equivalente a $5 \%$ do peso vivo médio das rãs, com exceção do farelo de trigo e farelo de soja, que foi de $4 \%$ (CASTRO, 1996). Os alimentos foram oferecidos puros, com exceção do amido de milho que foi fornecido misturado com o fubá de milho na proporção de $20 \%$ de amido (BRAGA, 1996). Os alimentos foram umedecidos e fornecidos através de uma seringa descartável.

Concomitantemente, 40 animais na fase inicial e 28 na fase de terminação, distribuídos em três repetições, foram mantidos em jejum, por um período de 120 horas, sendo as últimas 72 horas para a coleta de excretas, para determinar as perdas endógenas e metabólicas, e assim calcular os valores de energia metabolizável verdadeira dos alimentos.

A coleta de excreta, juntamente com a água, foi efetuada duas vezes ao dia, por um período de 72 horas. Todo material coletado foi acondicionado em sacos plásticos identificados e congelados em freezer; logo após o material foi descongelado e pré-seco e levando à estufa ventilada com temperatura de $55^{\circ} \mathrm{C}$.

Posteriormente, foram realizadas as análises de matéria seca e proteína bruta, segundo a metodologia de SILVA (1990), no Laboratório de Nutrição Animal do CCA-UFES, e as análises de energia bruta, no Laboratório de Nutrição Animal do Departamento de Zootecnia da Universidade Federal de Viçosa.

\section{Resultados e Discussão}

Os valores de matéria seca aparentemente metabolizável (MSAM), energia metabolizável aparente (EMA) e energia metabolizável aparente corrigida pela retenção de nitrogênio (EMAn) dos ingredientes, para animais nas fases inicial de crescimento (imagos) e de terminação (adultos), encontram-se na Tabela 2.

Os valores de MSAM e EMA do fubá de milho determinados com os imagos e adultos foram, respectivamente, 6,88 e $25,18 \%$ menores que os encontrados por BRAGA et al (1998) e 7,84 e 18,86\% menores que os obtidos por CASTRO et al. (1998). Para os animais adultos foram encontrados valores 7,19 e 26,88\% menores que os dos imagos, os quais foram menores que os obtidos por BRAGA et al (1998) e14,12\% e 22,58 \% menores que os de CASTRO et al (1998). Os fatores que podem ter contribuído para estes resultados são a granulometria e a qualidade do fubá de milho utilizado.

Foi observado que o valor da digestibilidade do amido determinado com os animais na fase inicial de crescimento foi $47,24 \%$ inferior ao valor obtido com os animais na fase de terminação, com valores de MSAM passando de $49,60 \%$ para 73,03\%. Os valores de EMA variaram de $1553 \mathrm{kcal} / \mathrm{kg}$, na fase inicial, a $2132 \mathrm{kcal} / \mathrm{kg}$, na fase de terminação, o que proporcionou aumento de $37,28 \%$. Estes resultados foram inversos aos determinados por BRAGA et al (1998), os quais encontraram valores superiores de MSAM $(61,01 \%)$ e EMA $(2346 \mathrm{kcal} / \mathrm{kg})$, para os jovens, e valores bem inferiores para os adultos $(60,06 \%$ de MSAM e $2204 \mathrm{kcal} / \mathrm{kg}$ de EMA). Possivelmente, esta variação está em função da qualidade do próprio ingrediente utilizado, tanto do amido quanto do fubá de milho utilizado como veículo, que apresentou valores inferiores de digestibilidade para jovens e adultos, quando comparado com BRAGA et al. (1998) e CASTRO et al. (1998).

Foi observado que, entre os alimentos avaliados, o farelo de soja foi o que apresentou maior valor de EMA (2856 kcal/kg), determinado com os jovens, enquanto na determinação com adultos o farelo de 
Tabela 2 - Valores de matéria seca aparentemente metabolizável (MSAM), energia metabolizável aparente (EMA) e energia metabolizável aparente corrigida pela retenção de nitrogênio(EMAn)

Table 2 - Values of apparent metabolizable dry matter (AMDM - \%), apparent metabolizable energy (AME - kcal/kg) and apparent metabolizable energy corrected by the nitrogen retention (AMEn - $\mathrm{kcal} / \mathrm{kg})^{*}$

\begin{tabular}{|c|c|c|c|c|c|c|}
\hline \multirow[b]{2}{*}{$\begin{array}{l}\text { Ingrediente } \\
\text { Ingredient }\end{array}$} & \multicolumn{2}{|c|}{$\begin{array}{l}\operatorname{MSMA}(\%) \\
\operatorname{AMDM}(\%)\end{array}$} & \multicolumn{2}{|c|}{$\begin{array}{l}\mathrm{EMA}(\mathrm{kcal} / \mathrm{kg}) \\
A M E(k c a l / k g)\end{array}$} & \multicolumn{2}{|c|}{$\begin{array}{l}\text { EMAn }(\mathrm{kcal} / \mathrm{kg}) \\
\text { AMEn }(\text { kcal/kg) }\end{array}$} \\
\hline & $\begin{array}{l}\text { Inicial } \\
\text { Initial }\end{array}$ & $\begin{array}{l}\text { Adultos } \\
\text { Adult }\end{array}$ & $\begin{array}{l}\text { Inicial } \\
\text { Initial }\end{array}$ & $\begin{array}{l}\text { Adultos } \\
\text { Adult }\end{array}$ & $\begin{array}{l}\text { Inicial } \\
\text { Initial }\end{array}$ & $\begin{array}{l}\text { Adultos } \\
\text { Adult }\end{array}$ \\
\hline $\begin{array}{l}\text { Fubá de milho } \\
\text { Corn meal }\end{array}$ & 55,70 & 58,44 & 1842 & 1983 & 1798 & 1893 \\
\hline $\begin{array}{l}\text { Amido de milho } \\
\text { Corn starch }\end{array}$ & 49,60 & 73,03 & 1553 & 2132 & 1524 & 2085 \\
\hline $\begin{array}{l}\text { Farelo de soja } \\
\text { Soybean meal }\end{array}$ & 76,97 & 71,50 & 2856 & 2718 & 2405 & 2284 \\
\hline $\begin{array}{l}\text { Farelo de trigo } \\
\text { Wheat middling }\end{array}$ & 35,95 & 87,54 & 1192 & 2800 & 1097 & 2644 \\
\hline $\begin{array}{l}\text { Farinha de peixe } \\
\text { Fish meal }\end{array}$ & 55,16 & 60,24 & 2288 & 2266 & 1915 & 1817 \\
\hline $\begin{array}{l}\text { Farinha de carne e ossos } \\
\text { Meat and bone meal }\end{array}$ & 29,63 & 59,01 & 1683 & 2080 & 1418 & 1835 \\
\hline
\end{tabular}

*Dados expressos na base da matéria seca (Data expressed in dry matter basis).

trigo foi o que apresentou maior valor de EMA $(2800 \mathrm{kcal} / \mathrm{kg})$. Já o farelo de soja apresentou valores cerca de 5,32\% maiores e o farelo de trigo, $13,25 \%$ maiores que os obtidos por CASTRO et al (1998).

Em geral, os resultados obtidos com rãs na fase de terminação foram superiores aos encontrados na fase inicial de crescimento, corroborando os resultados obtidos por CASTRO et al (1998) e BRAGA et al. (1998).

Observou-se que os resultados de MSAM do fubá de milho $(55,70 \%$ e $58,44 \%)$ obtidos, respectivamente, com os jovens e adultos, foram $10,95 \%$ maiores que os do amido $(49,60 \%$ e $73,06 \%)$, determinados com os jovens e $19,98 \%$ menores para os adultos, obtendo-se um resultado inverso ao encontrado por BRAGA et al (1998), mais uma vez mostrando a influência da qualidade do fubá de milho.

Os valores de EMAn foram, em média, respectivamente, para os jovens e para adultos, 95,93 e $96,70 \%$ menores que os valores de EMA para os ingredientes energéticos e 84,05 e $84,15 \%$ para os protéicos, o que corrobora os valores encontrados por CASTRO et al. (1998).

Quando se fez a correção de valores de EMA pela retenção de nitrogênio, verificou-se redução nestes valores. Estes resultados concordam com aqueles obtidos por CASTRO et al (1998) e BRAGA et al. (1998).

Os valores de energia metabolizável verdadeira (EMV), energia metabolizável verdadeira corrigida pela retenção de nitrogênio (EMVn) e a relação entre EMV e EMA, dos ingredientes, encontram-se na Tabela 3.

A correção nos valores de energia metabolizável verdadeira pela retenção de nitrogênio resultou em redução nos valores EMV, com média dos valores de EMVn correspondentes a 95,825 e 92,22\%, respectivamente, para os imagos e adultos, para os alimentos energéticos, e 84,30 e $78,96 \%$, para os alimentos protéicos.

Os valores de EMV foram, em média, 6,35 e $1,96 \%$ maiores que os valores EMA, respectivamente, para os imagos e adultos. Pode-se observar, portanto, que para os imagos a diferença foi maior, mostrando que o material endógeno excretado tem maior influência na fase inicial de crescimento. Os resultados obtidos com os adultos foram idênticos aos encontrados por CASTRO et al. (1998) e BRAGA et al. (1998).

Com exceção do fubá de milho, os resultados encontrados neste trabalho corroboram os valores encontrados por CASTRO et al. (1998) e BRAGA et al. (1998).

\section{Conclusões}

Os valores de matéria seca aparentemente metabolizável (MSAM - \%), energia metabolizável aparente (EMA - kcal/kg), energia metabolizável aparente corrigida pela retenção de nitrogênio (EMAn-kcal $/ \mathrm{kg}$ ), energia metabolizável verdadeira (EMV-kcal/kg) e energia metabolizável verdadeira corrigida pela retenção de nitrogênio (EMVn-kcal/kg) determinados para os diferentes ingredientes, para 
Rev. bras. zootec.

Tabela 3 - Valores de energia metabolizável verdadeira (EMV), energia metabolizável verdadeira corrigida pela retenção de nitrogênio (EMVn) e relação entre EMV e EMA dos ingredientes

Table 3 - Values of true metabolizable energy (TME), true corrected metabolizable energy by nitrogen retention (TMEn) and relation among $T M E$ and $A M E$ of the ingredients

\begin{tabular}{|c|c|c|c|c|c|c|}
\hline \multirow[b]{2}{*}{$\begin{array}{l}\text { Ingrediente } \\
\text { Ingredient }\end{array}$} & \multicolumn{2}{|c|}{$\begin{array}{l}\text { MSMA }(\%) \\
A M D M(\%)\end{array}$} & \multicolumn{2}{|c|}{$\begin{array}{l}\mathrm{EMA}(\mathrm{kcal} / \mathrm{kg}) \\
A M E(\mathrm{kcal} / \mathrm{kg})\end{array}$} & \multicolumn{2}{|c|}{$\begin{array}{l}\text { EMAn }(\mathrm{kcal} / \mathrm{kg}) \\
\text { AMEn }(\mathrm{kcal} / \mathrm{kg})\end{array}$} \\
\hline & $\begin{array}{r}\text { Inicial } \\
\text { Initial }\end{array}$ & $\begin{array}{l}\text { Adultos } \\
\text { Adults }\end{array}$ & $\begin{array}{r}\text { Inicial } \\
\text { Initial }\end{array}$ & $\begin{array}{l}\text { Adultos } \\
\text { Adults }\end{array}$ & $\begin{array}{l}\text { Inicial } \\
\text { Initial }\end{array}$ & $\begin{array}{l}\text { Adultos } \\
\text { Adults }\end{array}$ \\
\hline $\begin{array}{l}\text { Fubá de milho } \\
\text { Corn meal }\end{array}$ & 1948 & 1971 & 1896 & 1827 & 1,06 & 1,02 \\
\hline $\begin{array}{l}\text { Amido de milho } \\
\text { Corn starch }\end{array}$ & 1634 & 2175 & 1599 & 2007 & 1,05 & 1,02 \\
\hline $\begin{array}{l}\text { Farelo de soja } \\
\text { Soybean meal }\end{array}$ & 2965 & 2754 & 2508 & 2220 & 1,04 & 1,01 \\
\hline $\begin{array}{l}\text { Farelo de trigo } \\
\text { Wheat middling }\end{array}$ & 1361 & 2838 & 1256 & 2602 & 1,14 & 1,01 \\
\hline $\begin{array}{l}\text { Farinha de peixe } \\
\text { Fish meal }\end{array}$ & 2386 & 2347 & 1995 & 1672 & 1,04 & 1,04 \\
\hline $\begin{array}{l}\text { Farinha de carne e ossos } \\
\text { Meat and bone meal }\end{array}$ & 1765 & 2113 & 1495 & 1797 & 1,05 & 1,02 \\
\hline
\end{tabular}

*Dados expressos na base da matéria seca (Data expressed in dry matter basis).

os imagos, foram, respectivamente, de: fubá de milho, 55,70; 1842; 1798; 1948 e 1896; amido de milho, 49,60; 1553; 1524; 1634 e 1599; farelo de trigo, 35,$95 ; 1192 ; 1097 ; 1361$ e 1256 ; farelo de soja, 76,$97 ; 2856 ; 2405 ; 2965$ e 2508 ; farinha de peixe, 55,$16 ; 2288 ; 1915 ; 2386$ e 1995 ; e farinha de carne e ossos, 29,63; 1683; 1418; 1765 e 1495.

Para a fase de terminação os valores de MSAM (\%), EMA (kcal/kg), EMAn (kcal/kg), EMV $(\mathrm{kcal} / \mathrm{kg})$ e EMVn $(\mathrm{kcal} / \mathrm{kg})$ determinados para os diferentes ingredientes foram, respectivamente, de: fubá de milho, 58,44; 1934; 1893; 1971 e 1827 ; amido de milho, 73,03; ; 2132; 2085; 2175; 2007; farelo de trigo, 87,$54 ; 2800 ; 2644 ; 2838$ e 2602 ; farelo de soja, 71,$50 ; 2718 ; 2284 ; 2754$ e 2220 ; farinha de peixe, 60,$24 ; 2266 ; 1817 ; 2347$ e 1672 ; e farinha de carne e ossos, 59,01;2080; 1835; 2113 e 1797.

\section{Referências Bibliográficas}

BARBALHO, O.J.M. Exigência de proteína bruta de rã-touro (Rana catesbeiana Shaw, 1802), na fase de terminação. Viçosa, MG: UFV, 1991. 55p. Dissertação (Mestrado em Zootecnia) - Universidade Federal de Viçosa, 1991.

BRAGA, L.G.T. Valor nutritivo de alguns alimentos determinado para rã-touro (Rana catesbeiana Shaw, 1802) na fase de recria. Viçosa. UFV, 1996. 35p. Dissertação (Mestrado em Zootecnia) - Universidade Federal de Viçosa, 1996.

BRAGA, L.G.T., LIMA, S.L., DONZELE, J.L. et al. 1998. Valor nutritivo de alguns alimentos para rã - touro (Rana catesbeiana Shaw, 1802) na fase de recria. R. Bras. Zootec., 27(2):203-209.
CASTRO, J.C., LIMA, S.L., DONZELE, J.L. et al. Efeito da idade e do sexo da rã touro (Rana catesbeiana Shaw, 1802) na digestibilidade dos alimentos e determinação do período de jejum para realizar a alimentação forçada. In: INTERNATIONAL MEETING ON FROG RESEARCH AND TECHNOLOGY, 1, ENAR - Econtro Nacional de Ranicultura, 7, 1995, Viçosa - MG. Anais... Viçosa: ABETRA - UFV, 1995a. p.47.

CASTRO, J. C., LIMA, S. L., DONZELE, J. L. et al. Influência do número de refeições na taxa de passagem do alimento pelo trato digestivo da rã touro (Rana catesbeiana Shaw, 1802). In: INTERNATIONAL MEETING ON FROG RESEARCH AND TECHNOLOGY, 1, ENAR - Encontro Nacional de Ranicultura, 7, 1995, Viçosa - MG. Anais... Viçosa: ABETRA - UFV, 1995b. p.51.

CASTRO, J.C., LIMA, S.L., DONZELE, J.L. et al. Tempo de passagem do fubá de milho pelo trato digestivo da rã touro (Rana catesbeiana Shaw, 1802). In: INTERNATIONAL MEETING ON FROG RESEARCH AND TECHNOLOGY, 1, ENAR - Encontro Nacional de Ranicultura, 7, 1995, Viçosa - MG. Anais... Viçosa, ABETRA - UFV, 1995c. p.55.

CASTRO, J.C. Estrutura funcional do tubo digestivo e adaptação de uma metodologia para determinar os valores de energia metabolizável de alimentos para rã-touro (Rana catesbeiana Shaw, 1802). Viçosa, 1996. 120p. Tese (Doutorado em Zootecnia) - Universidade Federal de Viçosa, 1996.

CASTRO, J.C., LIMA, S.L., DONZELE, J.L. et al. 1998. Energia metabolizável de alguns alimentos usados em rações de rãs. R. Bras. Zootec., 27(2):203-209.

LIMA, S.L., AGOSTINHO, C.A. 1988. A criação de rãs. Rio de Janeiro: Globo. 187p. (Coleção do Agricultor - Pequenos Animais).

LIMA, S.L., AGOSTINHO, C.A. 1992. A tecnologia de criação de rãs. Viçosa: UFV. 168p.

MAZZONI, R., CARNEVIA, D., ROSSO, A. et al. Estudio del porcentaje de proteina y la energia en el alimento peleteado para engorde de rana toro (Rana catesbeiana Shaw, 1802). 
In: ENCONTRO NACIONAL DE RANICULTURA, 7, 1992, Rio de Janeiro. Anais... Rio de Janeiro: Associação de Ranicultores do Estado do Rio de Janeiro (ARERJ), 1992a. p.185-190.

MAZZONI, R., CARNEVIA, D., ROSSO, A. et al. Estudio del porcentaje de proteina en el alimento peleteado para engorde de rana toro (Rana catesbeiana Shaw, 1802). In: ENCONTRO NACIONAL DE RANICULTURA, 7, 1992, Rio de Janeiro. Anais... Rio de Janeiro: Associação de Ranicultores do Estado do Rio de Janeiro (ARERJ), 1992b. p.191-199.

MONTEIRO, E.S., LIMA, S.L., AGOSTINHO, C.A. Avaliação do desenvolvimento de rã-touro (Rana catesbeiana Shaw, 1802) alimentada com diferentes níveis de proteína. In: REUNIÃO ANUAL DA SOCIEDADE BRASILEIRA DE ZOOTECNIA, 25, 1988, Viçosa, MG. Anais... Viçosa: SBZ, 1988. p.77.

NATIONAL RESEARCH COUNCIL - NRC. 1983. Nutrient requirements of warmwater fishes and shellfishes, Washington, D.C. $102 \mathrm{p}$.

REEDER, W.G. 1964. The digestive sistem. In: Physiology of the anphibia. New York: Academic Press. 654p.

ROSTAGNO, H.S., SILVA, D.J., COSTA, P.M.A. et al. 1988. Composição de alimentos e exigências de aves e suinos (tabelas brasileiras). Viçosa, MG: UFV. 61p.
SILVA, D.J. 1990. Análise de alimentos (métodos químicos e biológicos ). 2 ed., Viçosa: UFV. 165p.

SIBBALD, I.R. 1976. A biossay for true metabolizable energy values measured with adult roosters. Poult. Sci., 54:303-308.

STÉFANI, M.V. Níveis de proteína adequada ao crescimento da rã-touro (Rana catesbeiana Shaw, 1802). In: INTERNATIONAL MEETING ON FROG RESEARCH AND TECHNOLOGY, 1, ENAR - Encontro Nacional de Ranicultura, 7, 1995, Viçosa - MG. Anais... Viçosa: ABETRA - UFV, 1995. p.65.

STÉFANI, M.V. Metabolismo e crescimento da rã touro (Rana catesbeiana Shaw, 1802) alimentada com niveis crescentes de carboidratos. Jaboticabal, 1996. 92p. Tese (Doutorado em Aquicultura) - Universidade Estadual Paulista, 1996.

Recebido em: 06/06/00

Aceito em: 09/11/00 